\title{
Interaction-free quantum computation
}

\author{
Hiroo Azuma* \\ Canon Inc., 5-1, Morinosato-Wakamiya, \\ Atsugi-shi, Kanagawa, 243-0193, Japan \\ E-mail: azuma.hiroo@canon.co.jp
}

March 23, 2004

\begin{abstract}
In this paper, we study the quantum computation realized by an interaction-free measurement (IFM). Using Kwiat et al.'s interferometer, we construct a two-qubit quantum gate that changes one particle's trajectory according to whether or not the other particle exists in the interferometer. We propose a method for distinguishing Bell-basis vectors, each of which consists of a pair of an electron and a positron, by this gate. (This is called the Bell-basis measurement.) This method succeeds with probability 1 in the limit of $N \rightarrow \infty$, where $N$ is the number of beam splitters in the interferometer. Moreover, we can carry out a controlled-NOT gate operation by the above Bell-basis measurement and the method proposed by Gottesman and Chuang. Therefore, we can prepare a universal set of quantum gates by the IFM. This means that we can execute any quantum algorithm by the IFM.
\end{abstract}

\section{Introduction}

Since many researchers recognized that a quantum computer solves certain problems more efficiently than any conventional computer, quantum computation has been studied eagerly [1]. The quantum computation is process of successive unitary transformations and measurements to two-state systems that are called qubits. Any unitary transformation applied to qubits can be decomposed into $U(2)$ transformations for one qubit and controlled-NOT (CNOT) gates for two qubits [2]. The CNOT gate generates entanglement between two qubits. Many researchers propose various methods for realizing the CNOT gate and carry out experiments 3 .

The Bell-basis measurement, which distinguishes Bell-basis vectors $\left\{\left|\Phi^{ \pm}\right\rangle,\left|\Psi^{ \pm}\right\rangle\right\}$, is a basic operation in quantum information processing. It plays an important role in the quantum teleportation 4. Gottesman and Chuang showed that we can carry out the CNOT gate if we prepare a certain special four-qubit state $|\chi\rangle$ and execute the Bell-basis

${ }^{*}$ On leave from Centre for Quantum Computation, Clarendon Laboratory, Parks Road, Oxford OX1 3PU, United Kingdom. 


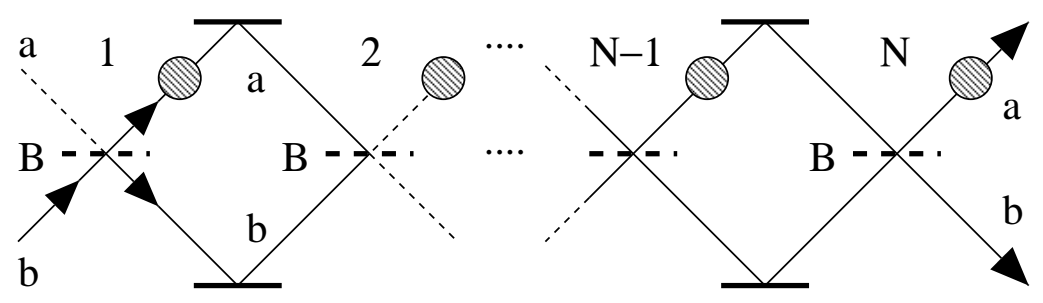

Figure 1: Kwiat et al.'s interferometer for the IFM.

measurement twice [5]. This implies the following fact. Let us assume that it is difficult to carry out the CNOT gate directly in a certain system. Even in this system, if we can accomplish the Bell-basis measurement easily, we can carry out the CNOT gate indirectly through Gottesman and Chuang's method.

In this paper, we consider the Bell-basis measurement realized by an interaction-free measurement (IFM). Using the IFM, we can construct a two-qubit gate that changes one particle's trajectory according to whether or not the other particle exists in the interferometer. By this two-qubit gate, we can generate the Bell states and a certain four-qubit state $|\chi\rangle$ introduced by Gottesman and Chuang [6, 7]. Hence, if we can carry out the Bell-basis measurement by the IFM, we can accomplish the CNOT gate indirectly. This is motivation of this paper.

This paper is organized as follows. In the latter half of this section, we review the IFM briefly. In Sec. 2, we consider the Bell-basis measurement by the IFM process. In Sec. 3, we discuss implementation of the CNOT gate by the IFM process. In Sec. 4, we give a brief discussion.

Here, we review the IFM. The IFM was proposed by Dicke, Elitzur, and Vaidman first [8, 9]. Then, it is refined by Kwiat et al. [10]. The IFM is a method for examining whether or not an object exists in an interferometer. We assume that the object absorbs a photon when the photon approaches the object closely enough. We have to detect the object without its absorption.

Kwiat et al. consider an interferometer that consists of $N$ beam splitters as shown in Fig. 1. We describe the upper paths as $a$ and lower paths as $b$, so that the beam splitters form the boundary line between the paths $a$ and the paths $b$ in the interferometer. We write a state with one photon on the paths $a$ as $|1\rangle_{a}$, and a state with no photon on the paths $a$ as $|0\rangle_{a}$. This notation applies to the paths $b$ as well. The beam splitter $B$ in Fig. 11 works as follows:

$$
B:\left\{\begin{array}{l}
|1\rangle_{a}|0\rangle_{b} \rightarrow \cos \theta|1\rangle_{a}|0\rangle_{b}-\sin \theta|0\rangle_{a}|1\rangle_{b} \\
|0\rangle_{a}|1\rangle_{b} \rightarrow \sin \theta|1\rangle_{a}|0\rangle_{b}+\cos \theta|0\rangle_{a}|1\rangle_{b}
\end{array}\right.
$$

(The transmissivity of $B$ is given by $T=\sin ^{2} \theta$, and the reflectivity of $B$ is given by $R=\cos ^{2} \theta$ in Eq. (11).)

Let us throw a photon into the lower left port of $b$ in Fig. 1 If there is no object on the paths, the wave function of the photon that comes from the $k$ th beam splitter is given by

$$
\sin k \theta|1\rangle_{a}|0\rangle_{b}+\cos k \theta|0\rangle_{a}|1\rangle_{b} \quad \text { for } k=0,1, \ldots, N
$$


If we assume $\theta=\pi / 2 N$, the photon that comes from the $N$ th beam splitter goes to the upper right port of $a$ with probability 1 .

Next, we consider the case where there is an object that absorbs the photon on the paths $a$. We assume that the object is put on every path $a$ that comes from each beam splitter, and all of these $N$ objects are the same one. The photon thrown into the lower left port of $b$ cannot go to the upper right port of $a$ because the object absorbs it. If the incident photon goes to the lower right port of $b$, it has not passed through paths $a$ in the interferometer.

Therefore, the probability that the photon goes to the lower right port of $b$ is equal to the product of the beam splitters' reflectivities. It is given by $P=\cos ^{2 N} \theta$. In the large $N$ limit, $P$ approaches 1 as follows:

$$
\lim _{N \rightarrow \infty} P=\lim _{N \rightarrow \infty} \cos ^{2 N}\left(\frac{\pi}{2 N}\right)=\lim _{N \rightarrow \infty}\left[1-\frac{\pi^{2}}{4 N}+O\left(\frac{1}{N^{2}}\right)\right]=1 .
$$

From the above discussion, we can conclude that Kwiat et al.'s interferometer directs an incident photon from the lower left port of $b$ with probability $P$ at least as follows: (1) if there is no absorptive object in the interferometer, the photon goes to the upper right port of $a ;(2)$ if there is the absorptive object in the interferometer, the photon goes to the lower right port of $b$. Furthermore, if we take large $N$, we can set $P$ arbitrarily close to 1 .

\section{The Bell-basis measurement by the IFM process}

Kwiat et al.'s IFM directs an outgoing photon from the interferometer to the ports of $a$ and $b$ in Fig. 1 according to whether or not an absorptive object exists on the paths $a$. It admits the interpretation that information about the object is written in the photon. Moreover, because there is no annihilation of the photon at the limit of $N \rightarrow \infty$ where $N$ is the number of beam splitters in the interferometer, reduction of the state does not occur during Kwiat et al.'s IFM. This means that there is no dissipation and the system retains coherence under the limit of $N \rightarrow \infty$.

From the above consideration, we can regard the absorptive object as quantum rather than classical. In Sec. 1, we consider the object to be a classical one that can take only one of two cases: the case where the object exists and the case where it does not exist. In this section, we treat the object as a quantum one and consider that it can take a superposition of the two orthogonal states where the object exists and does not exist.

From now on, we consider Kwiat et al.'s interferometer to be a kind of quantum gate as shown in Fig. 2] $a$ and $b$ in Fig. 20 correspond to the paths for the photon from the upper left and the lower left ports in Fig. [1 respectively. $a^{\prime}$ and $b^{\prime}$ in Fig. 2 correspond to the paths for the photon from the upper right and the lower right ports in Fig. 1 1 respectively, as well. The absorptive object comes from the path $x$ and goes to the path $x^{\prime}$ in Fig. [2] If we put the object into the path $x$ and put the photon into the path $a$ in Fig. [2 at the same time, the dissipation occurs in the system. Hence, we put a black rectangle on the port of $a$ in the gate drawn in Fig. 2 From now on, we call the symbol of Fig. 2 the IFM gate. Sometimes we call paths $x$ and $x^{\prime}$ control parts and $a, b, a^{\prime}$, and $b^{\prime}$ target parts in the IFM gate. 


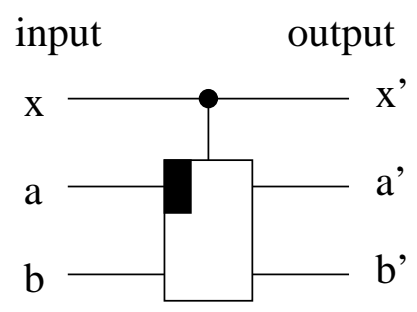

Figure 2: The IFM gate that represents Kwiat et al.'s interferometer.

\begin{tabular}{|c|c|}
\hline input & output \\
\hline$|0\rangle_{x}|0\rangle_{a}|1\rangle_{b}$ & $|0\rangle_{x}|1\rangle_{a}|0\rangle_{b}$ \\
$|1\rangle_{x}|0\rangle_{a}|1\rangle_{b}$ & $|1\rangle_{x}|0\rangle_{a}|1\rangle_{b}$ \\
$|0\rangle_{x}|1\rangle_{a}|0\rangle_{b}$ & $-|0\rangle_{x}|0\rangle_{a}|1\rangle_{b}$ \\
$|1\rangle_{x}|0\rangle_{a}|0\rangle_{b}$ & $|1\rangle_{x}|0\rangle_{a}|0\rangle_{b}$ \\
\hline
\end{tabular}

Table 1: The function of the IFM gate under the limit of $N \rightarrow \infty$.

Table 1 shows how the IFM gate transforms states under the limit of $N \rightarrow \infty$. The first line of Table 1 corresponds to a case where the absorptive object is not thrown into the path $x$ and the photon is thrown into the path $b$. The second line of Table 1 corresponds to a case where the absorptive object and the photon are incident into the path $x$ and the path $b$ respectively. We pay attention to the following fact. When we do not put the object into the path $x$ and put the photon into the path $a$, the wave function is multiplied by a phase factor $(-1)$ as the third line of Table 1. If we throw the particles into the paths $x$ and $a$ at the same time, the object absorbs the photon and the dissipation occurs. Thus the IFM gate is not unitary. From now on, when we think about the IFM gate, we take the limit of $N \rightarrow \infty$ and assume that the transformation of Table 1 is valid.

To put the following discussion concretely, we replace the photon with an electron $e^{-}$and replace the object with a positron $e^{+}$. If the electron and the positron approach each other closely enough, we assume that they are annihilated and a photon is created with probability 1 . We consider this phenomenon to be the absorption of the electron by the positron. Because the electron and the positron are quantum particles, we have to regard them as wave packets whose fluctuations are given by $|\Delta \boldsymbol{x}||\Delta \boldsymbol{p}| \sim \hbar / 2$. We suppose that $\Delta r$ is a characteristic length of the interaction between the electron and the positron. That is, the distance between them has to be less than $\Delta r$ for their annihilation. In our discussion, we assume $|\Delta \boldsymbol{x}| \ll \Delta r$. Therefore, we can consider the electron and the positron to be point particles. We can make beam splitters and mirrors for the electron and the positron from plates that have suitable potential barriers. We can adjust a reflectivity, a transmissivity, and a phase shift of the beam splitter by its potential barrier.

In this paper, we construct the logical ket vectors $\{|\overline{0}\rangle,|\overline{1}\rangle\}$ of a qubit from two paths $a$ and $b$. Hence, only one particle must always be on either of the two paths as $|\overline{0}\rangle=$ $|0\rangle_{a}|1\rangle_{b}$ and $|\overline{1}\rangle=|1\rangle_{a}|0\rangle_{b}$. This method for constructing a qubit is called the dual-rail 


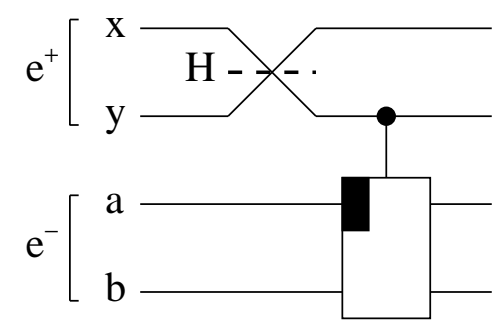

Figure 3: A quantum circuit for generating the Bell state of an electron and a positron.

representation [11].

The simplest usage of the IFM gate is the generation of the Bell state as shown in Fig. 3. $H$ is a beam splitter that works as follows:

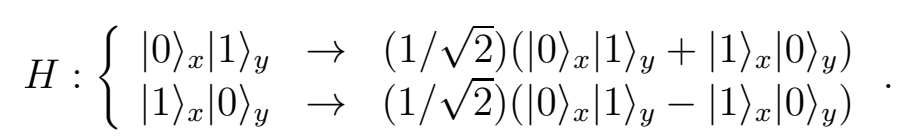

We put $|\overline{0}\rangle_{+}|\overline{0}\rangle_{-}=|0\rangle_{x}|1\rangle_{y}|0\rangle_{a}|1\rangle_{b}$ in a quantum circuit shown in Fig. 3 as an initial state. In this circuit, the positron goes along the paths $x, y$ and the electron goes along the paths $a, b$. From Eq. (4) and Table 1, a state in the circuit of Fig. 3] varies as follows:

$$
\begin{array}{ll} 
& |\overline{0}\rangle_{+}|\overline{0}\rangle_{-}=|0\rangle_{x}|1\rangle_{y}|0\rangle_{a}|1\rangle_{b} \\
\stackrel{H}{\longrightarrow} & (1 / \sqrt{2})\left(|0\rangle_{x}|1\rangle_{y}+|1\rangle_{x}|0\rangle_{y}\right)|0\rangle_{a}|1\rangle_{b} \\
\stackrel{\text { IFM }}{\longrightarrow} & (1 / \sqrt{2})\left(|0\rangle_{x}|1\rangle_{y}|0\rangle_{a}|1\rangle_{b}+|1\rangle_{x}|0\rangle_{y}|1\rangle_{a}|0\rangle_{b}\right) \\
& =(1 / \sqrt{2})\left(|\overline{0}\rangle_{+}|\overline{0}\rangle_{-}+|\overline{1}\rangle_{+}|\overline{1}\rangle_{-}\right)=\left|\Phi^{+}\right\rangle .
\end{array}
$$

Hence, the quantum circuit shown in Fig. 3 generates the Bell state $\left|\Phi^{+}\right\rangle$.

The beam splitter $H$ in Fig. 3 applies the Hadamard transformation to the qubit represented by the positron on the paths $x$ and $y$. Eq. (4) causes the following transformation:

$$
\left\{\begin{array}{lll}
|\overline{0}\rangle_{+} & \rightarrow & (1 / \sqrt{2})\left(|\overline{0}\rangle_{+}+|\overline{1}\rangle_{+}\right) \\
|\overline{1}\rangle_{+} & \rightarrow & (1 / \sqrt{2})\left(|\overline{0}\rangle_{+}-|\overline{1}\rangle_{+}\right)
\end{array}\right.
$$

As shown above, by using a beam splitter, we can realize an arbitrary $U(2)$ transformation applied to one qubit that is given by the dual-rail representation.

Then, let us consider the Bell-basis measurement by the IFM gate. Here, we consider the Bell states made of the electron and the positron as

$$
\begin{aligned}
\left|\Phi^{ \pm}\right\rangle & =(1 / \sqrt{2})\left(|\overline{0}\rangle_{+}|\overline{0}\rangle_{-} \pm|\overline{1}\rangle_{+}|\overline{1}\rangle_{-}\right) \\
\left|\Psi^{ \pm}\right\rangle & =(1 / \sqrt{2})\left(|\overline{0}\rangle_{+}|\overline{1}\rangle_{-} \pm|\overline{1}\rangle_{+}|\overline{0}\rangle_{-}\right)
\end{aligned}
$$

where $|\overline{0}\rangle_{+}=|0\rangle_{a}|1\rangle_{b},|\overline{1}\rangle_{+}=|1\rangle_{a}|0\rangle_{b},|\overline{0}\rangle_{-}=|0\rangle_{c}|1\rangle_{d},|\overline{1}\rangle_{-}=|1\rangle_{c}|0\rangle_{d}$, the positron goes along the paths $a, b$, and the electron goes along the paths $c, d$. A quantum circuit shown in Fig. 4 distinguishes these Bell states $\left\{\left|\Phi^{ \pm}\right\rangle,\left|\Psi^{ \pm}\right\rangle\right\}$.

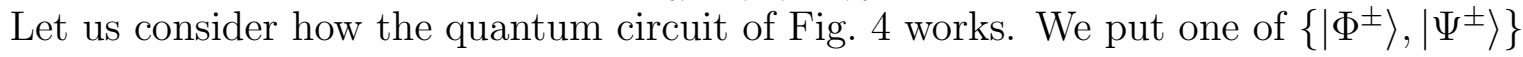
into the paths $a, b, c$, and $d$ at $t=0$. Table 2 shows how the quantum circuit of Fig. 4 


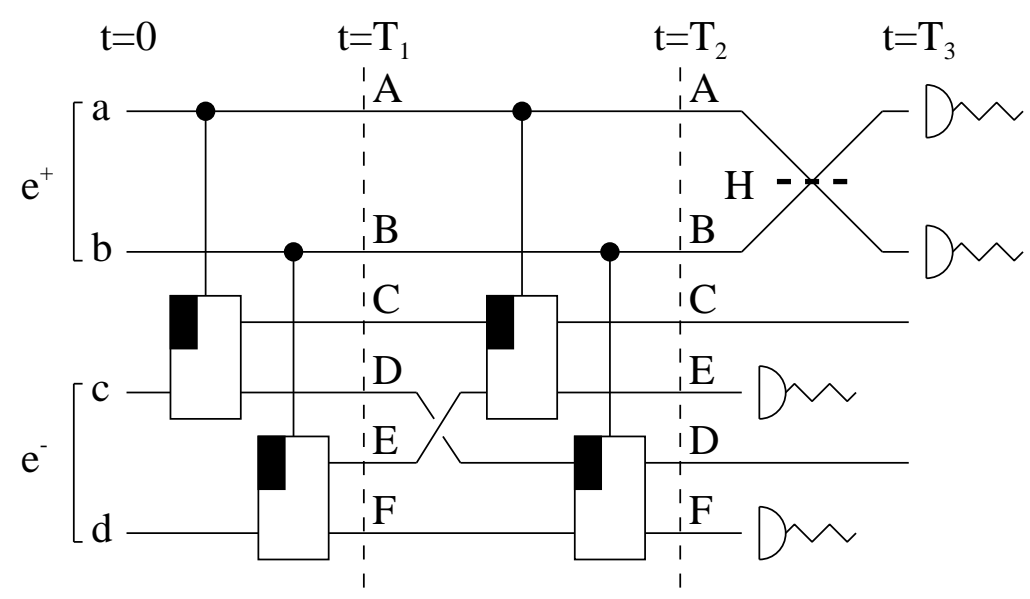

Figure 4: A quantum circuit for the Bell-basis measurement.

\begin{tabular}{|c|c|c|}
\hline$t=0$ & $t=T_{1}$ & $t=T_{2}$ \\
\hline$|0\rangle_{a}|1\rangle_{b}|0\rangle_{c}|1\rangle_{d}$ & $|0\rangle_{A}|1\rangle_{B}|0\rangle_{C}|0\rangle_{D}|0\rangle_{E}|1\rangle_{F}$ & $|0\rangle_{A}|1\rangle_{B}|0\rangle_{C}|0\rangle_{E}|0\rangle_{D}|1\rangle_{F}$ \\
$|0\rangle_{a}|1\rangle_{b}|1\rangle_{C}|0\rangle_{d}$ & $|0\rangle_{A}|1\rangle_{B}|1\rangle_{C}|0\rangle_{D}|0\rangle_{E}|0\rangle_{F}$ & $-|0\rangle_{A}|1\rangle_{B}|0\rangle_{C}|1\rangle_{E}|0\rangle_{D}|0\rangle_{F}$ \\
$|1\rangle_{a}|0\rangle_{b}|0\rangle_{c}|1\rangle_{d}$ & $|1\rangle_{A}|0\rangle_{B}|0\rangle_{C}|0\rangle_{D}|1\rangle_{E}|0\rangle_{F}$ & $|1\rangle_{A}|0\rangle_{B}|0\rangle_{C}|1\rangle_{E}|0\rangle_{D}|0\rangle_{F}$ \\
$|1\rangle_{a}|0\rangle_{b}|1\rangle_{c}|0\rangle_{d}$ & $|1\rangle_{A}|0\rangle_{B}|0\rangle_{C}|1\rangle_{D}|0\rangle_{E}|0\rangle_{F}$ & $-|1\rangle_{A}|0\rangle_{B}|0\rangle_{C}|0\rangle_{E}|0\rangle_{D}|1\rangle_{F}$ \\
\hline
\end{tabular}

Table 2: Variation of states in Fig. 团at $t=T_{1}$ and $t=T_{2}$.

transforms the basis vectors at $t=T_{1}$ and $t=T_{2}$. The basis vectors are given by $|\overline{0}\rangle_{+}|\overline{0}\rangle_{-}$, $|\overline{0}\rangle_{+}|\overline{1}\rangle_{-},|\overline{1}\rangle_{+}|\overline{0}\rangle_{-}$, and $|\overline{1}\rangle_{+}|\overline{1}\rangle_{-}$at $t=0$ as initial states. Here we note that the path $D$ and the path $E$ are exchanged between $t=T_{1}$ and $t=T_{2}$. We observe the electron on the paths $E$ and $F$ at $t=T_{2}$. If we detect the electron on the path $E$, we become aware that the initial state is $\left|\Psi^{ \pm}\right\rangle$. On the other hand, if we detect the electron on the path $F$, we become aware that the initial state is $\left|\Phi^{ \pm}\right\rangle$. Because the electron is neither on the path $C$ nor on the path $D$ and the state is always $|0\rangle_{C}|0\rangle_{D}$, we neglect these paths.

If we detect the electron on the path $E$, the initial state $\left|\Psi^{ \pm}\right\rangle$is projected onto the following state at $t=T_{2}$ :

$$
(1 / \sqrt{2})\left(-|0\rangle_{A}|1\rangle_{B} \pm|1\rangle_{A}|0\rangle_{B}\right) .
$$

The function of the beam splitter $H$ is defined by Eq. (44). Hence, $H$ transforms Eq. (8) as follows:

$$
\stackrel{H}{\longrightarrow}\left\{\begin{array}{ll}
-|1\rangle_{A}|0\rangle_{B} & \text { for }\left|\Psi^{+}\right\rangle \\
-|0\rangle_{A}|1\rangle_{B} & \text { for }\left|\Psi^{-}\right\rangle
\end{array} .\right.
$$

Therefore, if we detect the positron on the path $A$ at $t=T_{3}$, we find that the initial state is $\left|\Psi^{+}\right\rangle$, and if we detect the positron on the path $B$, we find that the initial state is $\left|\Psi^{-}\right\rangle$.

Next, we consider the case where the electron is detected on the path $F$. In this case, the initial state $\left|\Phi^{ \pm}\right\rangle$is projected onto the following state at $t=T_{2}$ :

$$
(1 / \sqrt{2})\left(|0\rangle_{A}|1\rangle_{B} \mp|1\rangle_{A}|0\rangle_{B}\right) .
$$


The beam splitter $H$ transforms Eq. (10) as follows:

$$
\stackrel{H}{\longrightarrow}\left\{\begin{array}{ll}
|1\rangle_{A}|0\rangle_{B} & \text { for }\left|\Phi^{+}\right\rangle \\
|0\rangle_{A}|1\rangle_{B} & \text { for }\left|\Phi^{-}\right\rangle
\end{array} .\right.
$$

Therefore, if we detect the positron on the path $A$ at $t=T_{3}$, we find that the initial state is $\left|\Phi^{+}\right\rangle$, and if we detect the positron on the path $B$, we find that the initial state is $\left|\Phi^{-}\right\rangle$. From the above discussion, we can distinguish the Bell basis vectors $\left\{\left|\Phi^{ \pm}\right\rangle,\left|\Psi^{ \pm}\right\rangle\right\}$by the IFM process.

\section{The CNOT gate operation by the IFM process}

Gottesman and Chuang showed that we can construct the CNOT gate by preparing a state

$$
|\chi\rangle=(1 / 2)[(|00\rangle+|11\rangle)|00\rangle+(|01\rangle+|10\rangle)|11\rangle]
$$

executing the Bell-basis measurement twice, and carrying out one-qubit gate operations according to results of the Bell-basis measurement [5]. Hence, we consider a method for generating $|\chi\rangle$ here.

Fig. [5 shows a quantum circuit for generating $|\chi\rangle$. We prepare $|\overline{0}\rangle_{+}|\overline{0}\rangle_{-}|\overline{0}\rangle_{+}|\overline{0}\rangle_{-}$as an initial state. In Fig. 5 , the state varies as follows:

$$
\begin{aligned}
& |\overline{0}\rangle_{+}|\overline{0}\rangle_{-}|\overline{0}\rangle_{+}|\overline{0}\rangle_{-} \\
\stackrel{H}{\longrightarrow} & (1 / \sqrt{2})\left(|\overline{0}\rangle_{+}+|\overline{1}\rangle_{+}\right)|\overline{0}\rangle_{-}|\overline{0}\rangle_{+}|\overline{0}\rangle_{-} \\
\stackrel{\mathrm{IFM} 1}{\longrightarrow} & (1 / \sqrt{2})\left(|\overline{0}\rangle_{+}|\overline{0}\rangle_{-}+|\overline{1}\rangle_{+}|\overline{1}\rangle_{-}\right)|\overline{0}\rangle_{+}|\overline{0}\rangle_{-} \\
\stackrel{\mathrm{IFM} 2}{\longrightarrow} & (1 / \sqrt{2})\left(|\overline{0}\rangle_{+}|\overline{0}\rangle_{-}|\overline{0}\rangle_{+}+|\overline{1}\rangle_{+}|\overline{1}\rangle_{-}|\overline{1}\rangle_{+}\right)|\overline{0}\rangle_{-} \\
\stackrel{\mathrm{H} \otimes}{\longrightarrow} H & (1 / 2)\left[\left(|\overline{0}\rangle_{+}|\overline{0}\rangle_{-}+|\overline{1}\rangle_{+}|\overline{1}\rangle_{-}\right)|\overline{0}\rangle_{+}+\left(|\overline{0}\rangle_{+}|\overline{1}\rangle_{-}+|\overline{1}\rangle_{+}|\overline{0}\rangle_{-}\right)|\overline{1}\rangle_{+}\right]|\overline{0}\rangle_{-} \\
\stackrel{\mathrm{IFM} 3}{\longrightarrow} & (1 / 2)\left[\left(|\overline{0}\rangle_{+}|\overline{0}\rangle_{-}+|\overline{1}\rangle_{+}|\overline{1}\rangle_{-}\right)|\overline{0}\rangle_{+}|\overline{0}\rangle_{-}+\left(|\overline{0}\rangle_{+}|\overline{1}\rangle_{-}+|\overline{1}\rangle_{+}|\overline{0}\rangle_{-}\right)|\overline{1}\rangle_{+}|\overline{1}\rangle_{-}\right] \\
& =|\chi\rangle .
\end{aligned}
$$

Here we use the following fact which can be derived from Table 1 . The IFM gate causes a transformation as $|\overline{0}\rangle_{+}|\overline{0}\rangle_{-} \rightarrow|\overline{0}\rangle_{+}|\overline{0}\rangle_{-}$and $|\overline{1}\rangle_{+}|\overline{0}\rangle_{-} \rightarrow|\overline{1}\rangle_{+}|\overline{1}\rangle_{-}$when the positron is a control qubit and the electron is a target qubit. As shown above, we can generate $|\chi\rangle$.

Fig. [6 shows a method for constructing the CNOT gate proposed by Gottesman and Chuang. A thin line transmits a qubit, and a thick line transmits a classical bit. (In Figs. 2, 3, 4, and 5] a horizontal line of quantum circuits represents a path along which a particle runs.) $B_{1}$ and $B_{2}$ stand for the Bell-basis measurements. An output of $B_{i}$ $(i \in\{1,2\})$ is given by two classical bits: $\left(x_{i}, z_{i}\right)=(0,0)$ for $\left|\Phi^{+}\right\rangle,(0,1)$ for $\left|\Phi^{-}\right\rangle,(1,0)$ for $\left|\Psi^{+}\right\rangle$, and $(1,1)$ for $\left|\Psi^{-}\right\rangle$. We apply $\sigma_{x}$ for $x_{i}=1$ and do nothing for $x_{i}=0$. We apply $\sigma_{z}$ for $z_{i}=1$ and do nothing for $z_{i}=0$ as well. We note that we can carry out any one-qubit unitary transformation, as $\sigma_{x}$ and $\sigma_{z}$, by a beam splitter.

From the above discussion, we find that we can apply the CNOT gate to an arbitrary state of an electron $\forall|\psi\rangle_{-}$and an arbitrary state of a positron $\forall|\phi\rangle_{+}$using the IFM gates and beam splitters. Then we have the following question. How do we apply the CNOT 


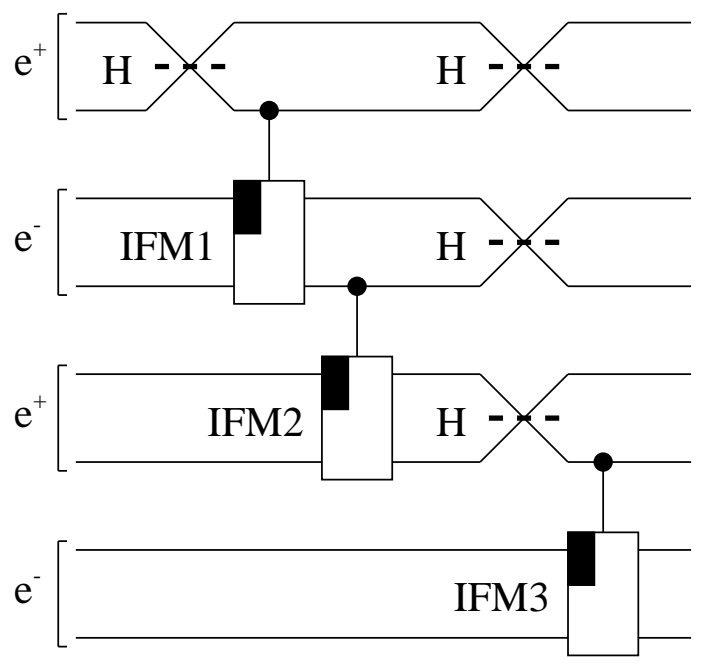

Figure 5: A quantum circuit for generating $|\chi\rangle$.

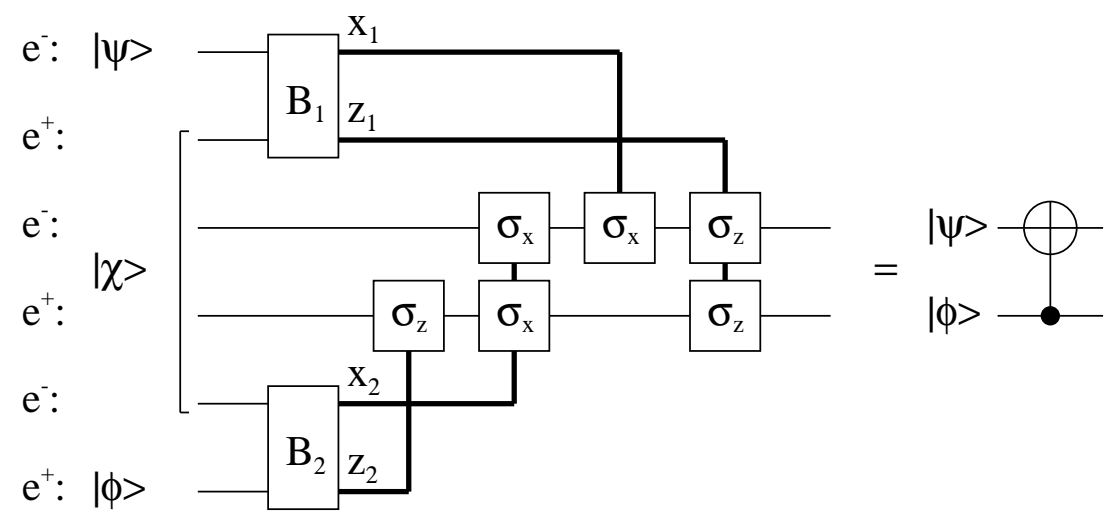

Figure 6: Gottesman and Chuang's method for constructing the CNOT gate by the Bellbasis measurement. A thin line transmits a qubit, and a thick line transmits a classical bit. 


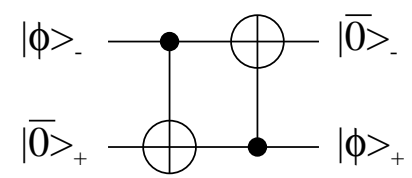

Figure 7: A quantum circuit for exchanging wave functions of the electron and the positron. A horizontal line represents a qubit.

gate to two arbitrary states of electrons $\forall|\psi\rangle_{-}$and $\forall|\phi\rangle_{-}$? To solve this problem, we use a technique shown in Fig. 7. (In Fig. [7, a horizontal line represents a qubit like Fig. 6.) We apply the CNOT gate twice as shown in Fig. 7 to the state of the electron $|\phi\rangle_{-}$and an auxiliary state of a positron $|\overline{0}\rangle_{+}$. Then, the wave functions are exchanged and we obtain $|\overline{0}\rangle_{-}$and $|\phi\rangle_{+}$. After these operations, we can apply the CNOT gate to $|\psi\rangle_{-}$and $|\phi\rangle_{+}$.

\section{Discussion}

If we make a qubit by the dual-rail representation, we can construct any one-qubit gate by a beam splitter. In this paper, we show that we can construct the CNOT gate by the IFM gates and beam splitters. These facts imply that we can prepare a universal set of quantum gates for quantum computation by the IFM. Therefore, we can consider the IFM to be a basic operation in the quantum information processing.

To carry out the Bell-basis measurement or to generate $|\chi\rangle$ with probability 1 , we need to take a limit of $N \rightarrow \infty$ where $N$ is the number of beam splitters in the interferometer of the IFM gate. In the limit of large $N$, the transmissivity of the beam splitter $T=$ $\sin ^{2}(\pi / 2 N)$ takes a very small value. This implies that we need many of highly accurate beam splitters to realize the IFM gate with high probability.

In this paper, we consider the IFM with an electron and a positron. To realize it, we need an accelerator. We may construct the IFM gate by a conductive electron and a hole in a semiconductor.

Horodecki investigated the dynamical evolution of the IFM process, where the absorptive object is replaced by an atom evolving coherently 12. Plenio et al. discussed the generation of entanglement between atoms inside an optical resonator through the non-detection of photons. This process resembles the IFM[13].

\section{Acknowledgements}

We thank M. Okuda for encouragement. We also thank M.B. Plenio for drawing our attention to Ref. [13].

\section{References}

[1] D. Deutsch and R. Jozsa, 'Rapid solution of problems by quantum computation', Proc. R. Soc. London, Ser. A 439, 553-558 (1992); 
D.R. Simon, 'On the power of quantum computation', SIAM J. Comput. 26, 1474$1483(1997)$

P.W. Shor, 'Polynomial-time algorithms for prime factorization and discrete logarithms on a quantum computer', SIAM J. Comput. 26, 1484-1509 (1997);

L.K. Grover, 'Quantum mechanics helps in searching for a needle in a haystack', Phys. Rev. Lett. 79, 325-328 (1997).

[2] A. Barenco, C.H. Bennett, R. Cleve, D.P. DiVincenzo, N. Margolus, P. Shor, T. Sleator, J.A. Smolin, and H. Weinfurter, 'Elementary gates for quantum computation', Phys. Rev. A 52, 3457-3467 (1995).

[3] Q.A. Turchette, C.J. Hood, W. Lange, H. Mabuchi, and H.J. Kimble, 'Measurement of conditional phase shifts for quantum logic', Phys. Rev. Lett. 75, 4710-4713 (1995); C. Monroe, D.M. Meekhof, B.E. King, W.M. Itano, and D.J. Wineland, 'Demonstration of a fundamental quantum logic gate', Phys. Rev. Lett. 75, 4714-4717 (1995);

E. Knill, R. Laflamme, and G.J. Milburn, 'A scheme for efficient quantum computation with linear optics', Nature (London) 409, 46-52 (2001);

T. Yamamoto, Yu.A. Pashkin, O. Astafiev, Y. Nakamura, and J.S. Tsuai, 'Demonstration of conditional gate operation using superconducting charge qubits', Nature (London) 425, 941-944 (2003).

[4] C.H. Bennett, G. Brassard, C. Crépeau, R. Jozsa, A. Peres, and W.K. Wootters, 'Teleporting an unknown quantum state via dual classical and Einstein-PodolskyRosen channels', Phys. Rev. Lett. 70, 1895-1899 (1993);

D. Bouwmeester, J.-W. Pan, K. Mattle, M. Eibl, H. Weinfurter, and A. Zeilinger, 'Experimental quantum teleportation', Nature (London) 390, 575-579 (1997).

[5] D. Gottesman and I.L. Chuang, 'Demonstrating the viability of universal quantum computation using teleportation and single-qubit operations', Nature (London) 402, 390-393 (1999).

[6] P.G. Kwiat, H. Weinfurter, A. Zeilinger, 'Interaction-free measurement of a quantum object: on the breeding of "Schrödinger cats" ', in Coherence and Quantum Optics VII, edited by J.H. Eberly, L. Mandel, and E. Wolf, (Plenum Press, New York, 1996), pp. 673 and 674 .

[7] H. Azuma, 'Interaction-free generation of entanglement', Phys. Rev. A 68, 022320 (2003).

[8] R.H. Dicke, 'Interaction-free quantum measurements: a paradox?', Am. J. Phys. 49, 925-930 (1981).

[9] A.C. Elitzur and L. Vaidman, 'Quantum mechanical interaction-free measurements', Found. Phys. 23, 987-997 (1993);

L. Vaidman, 'Are interaction-free measurements interaction free?', Opt. Spectrosc. 91, 352-357 (2001). 
[10] P. Kwiat, H. Weinfurter, T. Herzog, A. Zeilinger, and M.A. Kasevich, 'Interactionfree measurement', Phys. Rev. Lett. 74, 4763-4766 (1995);

P.G. Kwiat, A.G. White, J.R. Mitchell, O. Nairz, G. Weihs, H. Weinfurter, and A. Zeilinger, 'High-efficiency quantum interrogation measurements via the quantum Zeno effect', Phys. Rev. Lett. 83, 4725-4728 (1999).

[11] I.L. Chuang and Y. Yamamoto, 'Simple quantum computer', Phys. Rev. A 52, 34893496 (1995).

[12] P. Horodecki, " "Interaction-free" interaction: entangling evolution coming from the possibility of detection', Phys. Rev. A 63, 022108 (2001).

[13] M.B. Plenio, S.F. Huelga, A. Beige, and P.L. Knight, 'Cavity-loss-induced generation of entangled atoms', Phys. Rev. A 59, 2468-2475 (1999). 